Volume 2, Issue 2, April-June 2017, Pages: 183, DOI: http://dx.doi.org/10.19082/ah183

\title{
MULTIPLE SCLEROSIS MINIMUM DATA SET
}

\author{
Golchehreh Ahmadi1*, Sima Ajami2, Sakineh Saghaeian-Nejad Isfahani3, Masoud Etemadifar4
}

1: MSc, Public Health Network of Shahin Shahr, Medical Education Research Center, Isfahan University of Medical Sciences, Isfahan, Iran.

2: Professor, Department of Management \& Health Information Technology, School of Medical Management and Information Sciences, Isfahan University of Medical Sciences, Isfahan, Iran.

3: Instructor, Social Determinant of Health Research Center, Isfahan University of Medical Sciences, Isfahan, Iran.

4: Professor, Isfahan Research Committee on Multiple Sclerosis, Isfahan, Iran.

\section{Correspondence:}

Golchehreh Ahmadi, Room No. 308, Second Floor, Educational Development Center, Isfahan University of Medical Sciences, Hezar Jerib Ave, Isfahan, Iran. Tel: +989378023823, E-mail: gc.ahmadi@gmail.com.

\section{TYPE OF ARTICLE: CONFERENCE ABSTRACT}

\begin{abstract}
Introduction: Absence of a standard information system for multiple sclerosis (MS) patients causes inaccuracy and unreliability when producing information and statistics about prevalence and incidence of MS in Iran. Minimum Data Set (MDS) is a standard tool for collecting and recording data, and the basis of information systems. The aim of this study was to establish Multiple Sclerosis Minimum Data Set (MSDMS) for Iran.

Methods: This research was an applied one and an exploratory study which was done in Isfahan, Iran, in four phases: 1) determining data elements related to MS in Iran and other countries; 2) extracting and categorizing data elements; 3) making an MSMDS draft; 4) evaluating of draft by Delphi technique. Ethics approval was not necessary. Data was analyzed by descriptive statistics indexes, (relative and absolute frequencies only).

Results: Following the results of 2 rounds Delphi technique, finally, MSMDS was established including 150 data elements in three categories: administrative, clinical and socioeconomic. Every category included, respectively, 6, 11 and 6 subcategories and 27, 84 and 39 data elements and detailed information.

Conclusion: The first and most important step to standardization of data collection nationally, is creating MDS. According to specialists' consensus on necessity of available data elements in presenting MSMDS, using this MDS is recommended as a basis for collecting data on MS patients.

KEYWORDS: Multiple Sclerosis, Information Systems, Long-Term Care Programs, Medical Record.
\end{abstract}

\footnotetext{
Abstracts of First National Congress of Medical Informatics, Mashhad, Iran, February 2017

(C) 2017 The Authors. This is an open access article under the terms of the Creative Commons Attribution-NonCommercialNoDerivs License, which permits use and distribution in any medium, provided the original work is properly cited, the use is non-commercial and no modifications or adaptations are made.
} 